\title{
Exploring the connectivity between the cerebellum and facial motor cortex •
}

\section{Dear Editor,}

Non-invasive stimulation has been used to study connectivity between cerebellum and primary motor cortex (M1). Ugawa and colleagues were the first to demonstrate that transcranial magnetic stimulation (TMS) delivered over the cerebellum 5-7 ms prior to a stimulus applied over the contralateral M1 reduced the excitability of M1 corticospinal outputs [1]. This phenomenon was termed cerebellar brain inhibition (CBI) [1-2] and has been interpreted as stimulation of Purkinje cells leading to inhibition of deep cerebellar nuclei which itself has an excitatory disynaptic connection to M1 [3].

While CBI has been shown to exist for hand [4-6] and leg muscle representations [6-7], it remains unknown whether $\mathrm{CBI}$ is present in regions of $\mathrm{M} 1$ that project to muscles of the face (fM1). In contrast to other body muscles, the muscles of the lower face receive a bilateral projection from fM1 [8], and therefore any cerebellar input might preferentially influence brainstem structures that receive input from both hemispheres rather than cortex. Since previous studies utilizing CBI have provided insights into the pathophysiology of disorders involving the cerebello-thalamo-M1 tracts [3], we tested whether we could detect any evidence for CBI-fM1 using conventional methodology.

\section{Methods}

Fourteen right-handed healthy volunteers ( 9 females; $28.77 \pm 1.11$ years old) participated in this study, conducted at University College of London (UCL). Informed written consent approved by the UCL ethics committee was obtained from all participants. None of them had a history of neurological symptoms or psychiatric disease, and none had any contraindications to TMS [10]. Participants sat in a comfortable chair and were asked to stay relaxed but alert during the experimental session.

Electromyographic activity (EMG) was recorded from the right depressor anguli oris muscle (DAO) as reported by Pilurzi et al. [8]. Unrectified EMG signals were recorded 
(D360 amplifier, Digitimer Ltd, Welwyn Garden City, UK), amplified (x1000), filtered (bandpass 3-3000 Hz), sampled (5 kHz/channel; window frame length: $250 \mathrm{~ms}$ ) using a power 1401 analog-to-digital converter (Cambridge Electronic Design, Cambridge, UK) and Signal 6.0 software on a computer and stored for off-line analysis.

TMS of fM1 was performed at the optimal spot using a figure-of-eight coil with external loop diameter of $7 \mathrm{~cm}$ connected to a Magstim 200 stimulator (Magstim Co., Whitland, Dyfed, UK) [8]. After determining the resting motor thresholds (RMT) for this muscle, the test stimulus (TS) intensity was set to evoke an MEP in the relaxed DAO of at a least a $0.3 \mathrm{mV}$ [10]. Cerebellar stimuli (CS) were delivered with a $90 \mathrm{~mm}$ diameter double cone coil connected to a Magstim 200 stimulator. We first tested whether cerebellar stimulation using an intensity of $70 \%$ of maximum stimulator output (MSO) was activating any corticobulbar output by testing for the presence of an MEP or a possible silent period in the DAO during strong contraction. If there was no sign of brainstem activation, the CS intensities were set to 50,60 and 70\% of MSO as reported in previous studies [2,6]. The experiment was divided into two sessions that were performed two different days: central and lateral CBI. When assessing central CBI, the CS was delivered at the inion, while the CS for lateral CBI was delivered $3 \mathrm{~cm}$ lateral to the inion contralateral to the stimulated M1 $[1,5]$.

In each session, 12 unconditioned and 12 conditioned MEPs were randomized, pairing CS over the cerebellum with TS over fM1 at either 5 or $7 \mathrm{~ms}$ interstimulus intervals (ISIs). Each intensity of CS was examined in a separate session. Amplitudes of conditioned and unconditioned responses were measured. CBI was expressed as the average MEP amplitude evoked by the cerebellar-conditioned stimulation of fM1 relative to the average MEP amplitude evoked by the unconditioned TMS pulses over fM1.

Statistical analysis was performed with SPSS 20 software (SPSS Inc, Chicago, IL, USA). Repeated measures (RM) analysis of variance (ANOVA) and planned post hoc ttest with Bonferroni correction for multiple comparison were used. Compound symmetry was evaluated with the Mauchly's test and the Greenhouse-Geisser correction was used when required. Significance was set for $p$ value $\leq 0.05$. Values are expressed as means \pm standard error of the mean (SEM). To investigate the presence of CBI, a two-way RM-ANOVA, on MEP amplitude obtained during lateral stimulation, with ISI 
(TS, 5 and $7 \mathrm{~ms}$ ), and INTENSITY (50\%, 60\%, 70\% of MSO) as within subject factors was used. To examine the effect of stimulation site a two-way RM-ANOVA on the ratio using a 5 ms ISI, with SITE (central or lateral CS) and INTENSITY (50\%, 60\%, 70\% of MSO) as within subject factors was performed.

\section{Results}

Mean RMT was 52.6 $\pm 2.9 \%$ MSO and the TS was $68.8 \pm 4.3 \%$ MSO. None of the subjects had a silent period in the active DAO following CS at 70\% MSO.

In the experiment using lateral stimulation a RM-ANOVA revealed a significant effect of ISI $\left(\mathrm{F}_{2,24}=3.403, \mathrm{p}=0.050\right)$ but not INTENSITY $\left(\mathrm{F}_{2,24}=0.471, \mathrm{p}=0.630\right)$ and no interaction among the factors $\left(\mathrm{F}_{4,48}=1.527, \mathrm{p}=0.233\right)$. Specifically, post hoc analysis showed that the conditioned MEP was significantly smaller than TS only at ISI $=5 \mathrm{~ms}$ $(\mathrm{p}=0.014)$, thus indicating a timing-specific effect of CBI on fM1 (Figure 1A).

When we compared the effect of stimulation site at 5ms ISI, a RM-ANOVA revealed a significant effect on CBI ratio of SITE $\left(\mathrm{F}_{2,24}=4.556, \mathrm{p}=0.054\right)$ and a non-significant trend for INTENSITY $\left(\mathrm{F}_{2,24}=2.681, \mathrm{p}=0.089\right)$. There was also a significant interaction $\left(\mathrm{F}_{2,24}=4.033, \mathrm{p}=0.038\right)$ term, indicating that the effect of intensity differed between sites. Bonferroni analysis demonstrated that CBI at $5 \mathrm{~ms}$ ISI was detectable only following lateral stimulation and a CS of $70 \%$ MSO ( $\mathrm{p}=0.005)$.

\section{Discussion}

We report the first evidence of cerebellar-M1 connectivity for the M1 representation of the DAO muscle in healthy subjects. Similar to CBI for the hand and leg representations, the timing and location of cerebellar stimulation prior to probing cortical excitability was critical suggesting that the same pathways targeted with paired-pulse TMS for limb M1 extends to the cerebellar connection with fM1.

Some limitations in this study have to be acknowledged such as its exploratory nature in a small sample size. Nevertheless. these preliminary data provide interesting insights into the understanding of the cerebellar control over facial muscles and may provide a useful tool to detect somatotopic-specific effects of fM1-CBI when individuals learn new tasks 
involving facial muscles. Finally, it may be useful in order to understand better the involvement of cerebellum in orofacial dystonia.

\section{Figures legend}

\section{Figure1. Cerebellar Brain Inhibition in face primary motor cortex}

A: Recordings of motor-evoked potentials (MEP) from the depressor anguli oris muscle (DAO) of a representative subject are reported for each condition (unconditioned MEP, induced by the test stimulus (TS), and conditioned MEPs at interstimulus intervals (ISIs) of 5 and $7 \mathrm{~ms}$ ) with a conditioning stimulus of $70 \%$ maximal stimulator output (MSO) applied over the right lateral cerebellum. B: Effects of the TS alone and of the paired CSTS at 5 and $7 \mathrm{~ms}$ ISIs and at different CS intensities on amplitude of the DAO MEP (mean \pm standard error, SE). The conditioned MEP at $5 \mathrm{~ms}$ was significantly smaller than

test MEP. C: Mean amplitude of conditioned DAO MEP (mean \pm SE, expressed as ratio of the unconditioned MEP) at $5 \mathrm{~ms}$ ISI and different intensities of the CS over the central and lateral cerebellum. ${ }^{*} \mathrm{p}<0.05$ 
[1] Ugawa Y, Uesaka Y, Terao Y, Hanajima R, Kanazawa I. Magnetic stimulation over the cerebellum in humans. Annals of Neurology 1995;37(6):703-13

[2] Galea JM, Jayaram G, Ajagbe L, Celnik P. Modulation of cerebellar excitability by polarityspecific noninvasive direct current stimulation. The Journal of neuroscience : the official journal of the Society for Neuroscience 2009;29(28):9115-22.

[3] Celnik P. Understanding and modulating motor learning with cerebellar stimulation. Cerebellum (London, England) 2015;14(2):171-4.

[4] Spampinato D, Celnik P. Temporal dynamics of cerebellar and motor cortex physiological processes during motor skill learning. Sci Rep 2017;7(40715).

[5] Pinto AD, Chen R. Suppression of the motor cortex by magnetic stimulation of the cerebellum. Experimental brain research 2001;140(4):505-10.

[6] Spampinato DA, Block HJ, Celnik PA. Cerebellar-M1 Connectivity Changes Associated with Motor Learning Are Somatotopic Specific. J Neurosci 2017;37(9):2377-86

[7] Jayaram G, Galea JM, Bastian AJ, Celnik P. Human locomotor adaptive learning is proportional to depression of cerebellar excitability. Cerebral cortex (New York, NY: 1991) 2011;21(8):1901-9.

[8] Pilurzi G, Hasan A, Saifee TA, Tolu E, Rothwell JC, Deriu F. Intracortical circuits, sensorimotor integration and plasticity in human motor cortical projections to muscles of the lower face. The Journal of physiology 2013;591(7):1889-906.

[9] Pilurzi G, Hasan A, Saifee TA, Tolu E, Rothwell JC, Deriu F. Intracortical circuits, sensorimotor integration and plasticity in human motor cortical projections to muscles of the lower face. The Journal of physiology 2013;591(7):1889-906.

[10] Rossini PM, Burke D, Chen R, Cohen LG, Daskalakis Z, Iorio R. Non-invasive electrical and magnetic stimulation of the brain, spinal cord, roots and peripheral nerves: Basic principles and procedures for routine clinical and research application. An updated report from an I.F.C.N. Committee. Clin Neurophysiol 2015;126. 